\title{
Direct Electrochemical Evaluation of Plasma Membrane Cholesterol in Live Mammalian Cells
}

\author{
Dechen Jiang ${ }^{1}$, Anando Devadoss ${ }^{1,4}$, M. Simona Palencsár ${ }^{1}$, Danjun Fang ${ }^{1}$, Nicole M. \\ White $^{2}$,Thomas J. Kelley ${ }^{2}$, Jonathan D. Smith ${ }^{3}$, James D. Burgess ${ }^{1 *}$ \\ ${ }^{1}$ Department of Chemistry, Case Western Reserve University, Cleveland, Ohio 44106, USA. \\ ${ }^{2}$ Departments of Pediatrics and Pharmacology, Case Western Reserve University and Rainbow \\ Babies and Children's Hospital, Cleveland, OH 44106, USA. ${ }^{3}$ Department of Cell Biology, \\ Cleveland Clinic, Cleveland, Ohio 44195, USA.
}

\section{Microelectrode detection of cholesterol in solution}

The modification of the electrode surface with cholesterol oxidase is conducted using the EDC/NHS coupling reaction (see below). ${ }^{1}$ The cholesterol oxidase modified microelectrodes are characterized for detection of cholesterol solution at $37^{\circ} \mathrm{C}$. Supporting Figure 1a shows representative amperometric responses for cholesterol solution $(25 \mu \mathrm{M}$ to $680 \mu \mathrm{M})$. Data from three replicate experiments is reflective of Michaelis-Menten enzyme kinetics $(\mathrm{Km}$ is $150 \mu \mathrm{M})$ as shown in Supporting Figure $1 \mathrm{~b}$. These data indicate that electrode-immobilized cholesterol oxidase retains activity for catalyzing the oxidation of cholesterol by molecular oxygen at physiological temperature. ${ }^{2}$ The electron transfer reaction scheme for cholesterol detection is shown in supporting scheme 1.

\section{Electrochemical measurements of plasma membrane cholesterol at the surface of single cells}

Supporting Figures 2 and 3 show all data used for construction of the bar graphs in Figures 2 and 3 of the communication. The data indicate that electrode responses for macrophages, AcLDL loaded macrophages, and AcLDL loaded macrophages with ACAT inhibitor are 42 - $58 \mathrm{fA}, 70$ - $90 \mathrm{fA}$, and 110 - $150 \mathrm{fA}$, respectively. Control experiments on the three cells types where performed using bare $\mathrm{Pt}$ microelectrodes containing no immobilized cholesterol oxidase (Supporting Figure 4). Bare Pt electrodes show no response or a small apparent reduction current. It is hypothesized that the negative shift in base line current observed for many of the control experiments reflects blocking of the bare $\mathrm{Pt}$ electrode surface (upon positioning the electrode in contact with the cell surface) from solution phase contaminants that are oxidized at the applied potential. No difference in the cell types is detected using bare Pt microelectrodes (see below; Supporting Figure 4). Because there is debate regarding the rate of cholesterol flip-flop between the plasma membrane leaflets (published $t_{1 / 2}$ values for cholesterol flip-flop range from seconds to days ${ }^{3)}$, we strictly state that the electrode response is a measure of the cholesterol content in the outer leaflet of the plasma membrane. We hypothesize, however, that the rate of cholesterol flip-flop is fast relative to the rate of cholesterol consumption at the contact site and that the measurements are a gauge of total plasma membrane cholesterol.

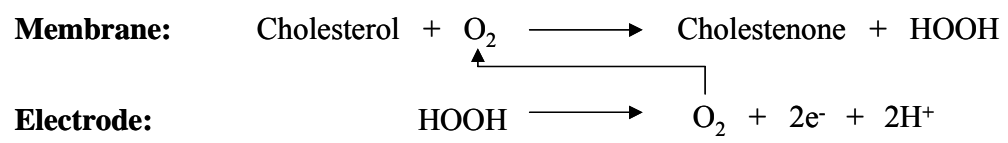

Supporting Scheme 1: In the membrane, the enzyme catalyzes the oxidation of cholesterol to cholestenone, and the reduction of molecular oxygen to hydrogen peroxide. At the electrode surface, hydrogen peroxide is electrochemically oxidized yielding oxygen and steady state current responses.

\footnotetext{
${ }^{4}$ Present address: Department of Chemistry, Stanford University, Stanford, California 94305, USA.
} 
Excessive contact experiments (e.g., 20 or more) conducted at the same cell can lead to altered electrode response. Most often, excessive contact experiments conducting using the same electrode and the same cell result in diminished electrode responses. While the reason for altered electrode response upon excessive cell contact experiments is unclear, the data presented here reflect a maximum of five contacts experiments per cell and a maximum of ten contact experiments per electrode (five contact experiments per cell type per electrode for each comparison).

There is ample cholesterol contained in the outer leaflet to produce the electrochemical responses observed. A calculation estimating the number of cholesterol molecules present in the region of the plasma membrane (outer leaflet only) that is directly adjacent to the $4 \mu \mathrm{m}$ diameter microelectrode (i.e., electrode footprint) gives 16 amoles assuming a cholesterol-to-phospholipid ratio of 0.33 in the plasma membrane. ${ }^{4}$ An electrode response of $\sim 50 \mathrm{fA}$ suggests that $\sim 15 \%$ of this amount of cholesterol is oxidized over $10 \mathrm{~s}$ (integrated current is equal to 3.2 pico-coulombs). It is estimated that ca. $4 \%$ of the total cholesterol contained in the outer leaflet of a macrophage $(\sim 8 \mu \mathrm{m}$ in diameter) is consumed during each contact experiment. Cholesterol is believed to be replenished to the electrode contact site between experiments by lateral diffusion of cholesterol in the plasma membrane during the several second interval between each contact of the electrode to the cell. Flip-flop (transbilayer movement) of cholesterol between the membrane leaflets, as well as delivery of cholesterol to the plasma membrane from stores inside the cell, are likely also involved in replenishment of cholesterol to the electrode contact site between contact experiments. While the electrode response correlates with plasma membrane cholesterol content, the measured (near steadystate) electrode responses could be controlled, in part, by the mass transfer rate for replenishment of cholesterol to the contact site.
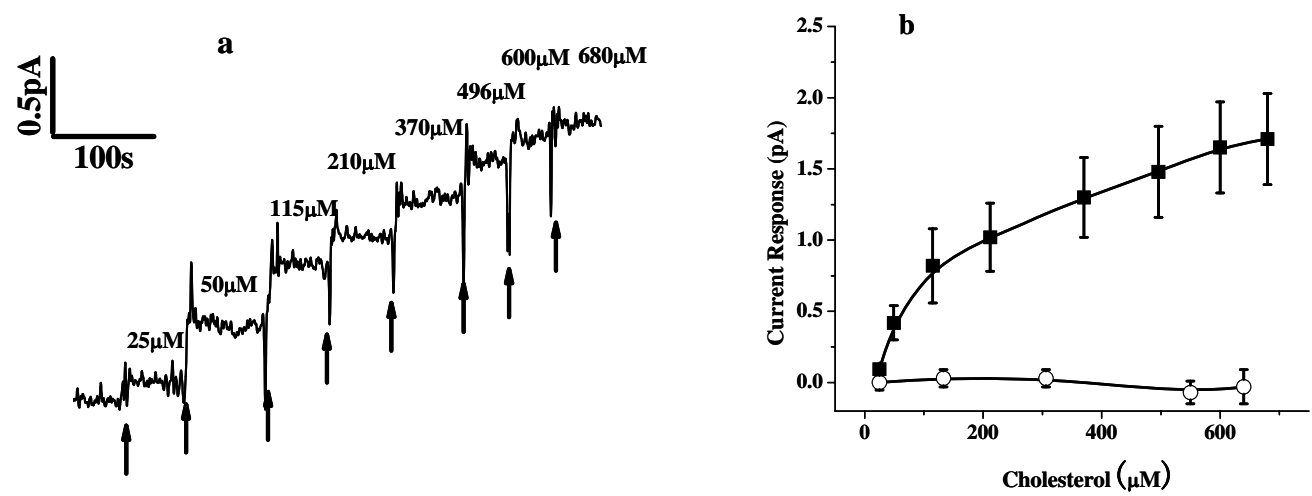

Supporting Figure 1: Characterization of cholesterol oxidase modified electrode (4 $\mu \mathrm{m}$ diameter) in quiet solution at $37^{\circ} \mathrm{C}$. (a) Representative amperometric responses for exposure to cholesterol solution. ( $\uparrow$ ) indicates the times for injecting cholesterol solution. (b) Plot of steady state current responses vs. cholesterol concentration for the oxidase modified microelectrodes (square) and bare Pt microelectrodes (circles). Error bars (mean $\pm \mathrm{SD}$ ) reflect three independent experiments using three different electrodes. Other conditions as stated in Supporting Methods. 

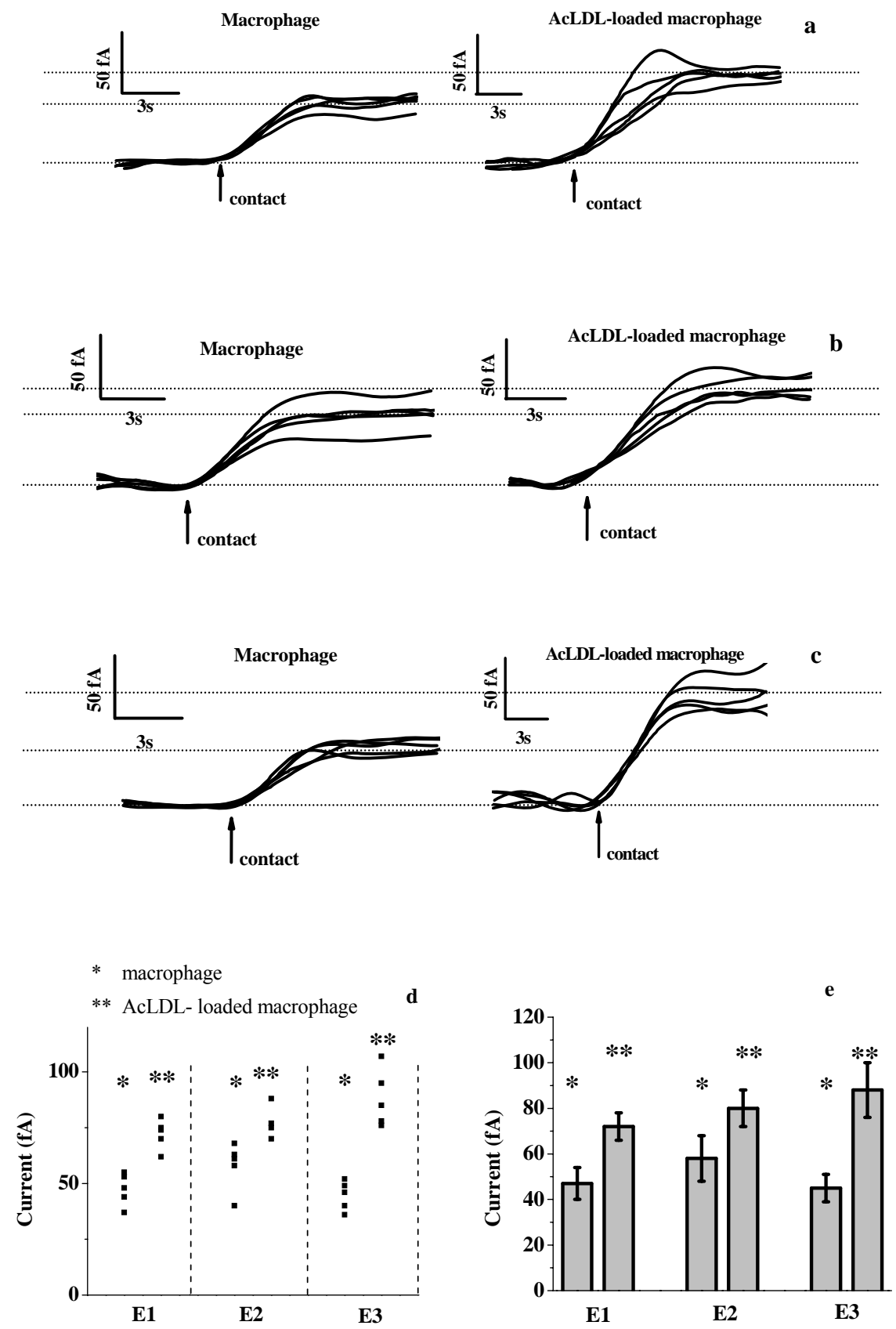

Supporting Figure 2: Microelectrode comparison of macrophages and AcLDL-loaded macrophages. (a) Electrode 1 (E1), (b) Electrode 2 (E2), (c) Electrode 3 (E3). (a-c) reflect three independent cell preparations and five sequential contact experiments per cell. The $(\uparrow)$ arrow indicates the time of contact between the electrode and cell plasma membrane. (d) Cluster plot showing all electrode responses. (e) Bar graph constructed for each electrode, error bars (mean \pm $\mathrm{SD})$ reflect five replicated contacts shown in a-c. 

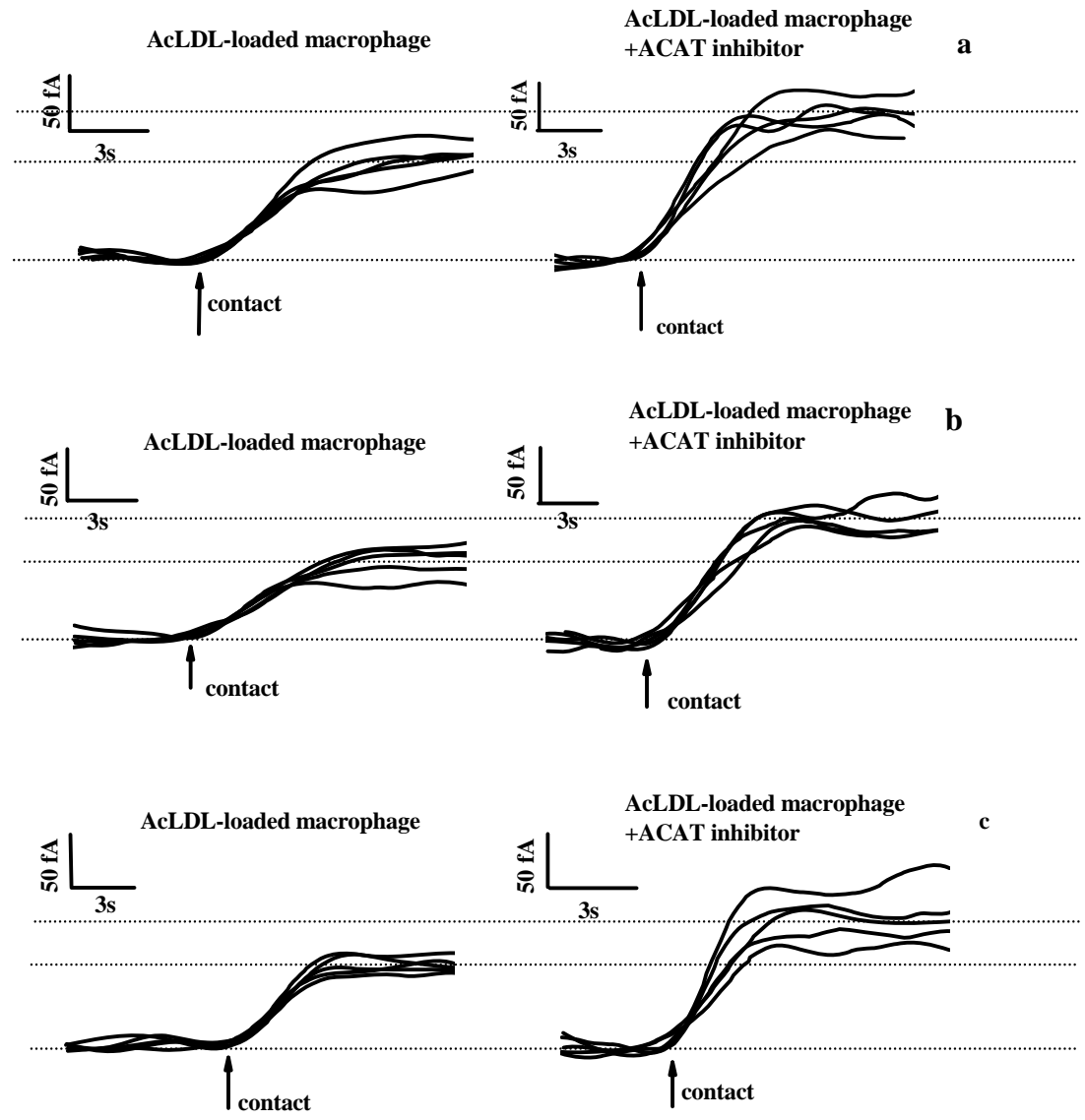

d

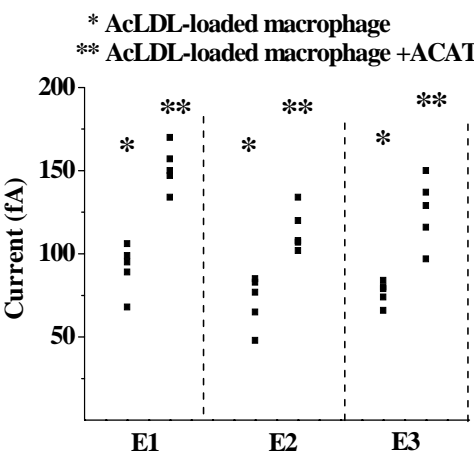

$\mathbf{e}$

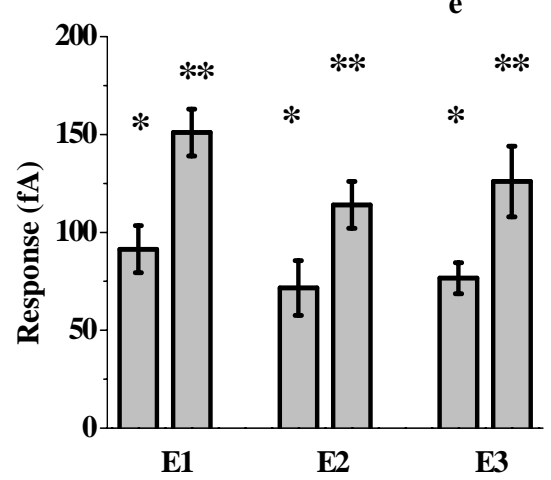

Supporting Figure 3: Microelectrode comparison of AcLDL-loaded macrophages and AcLDLloaded macrophages with ACAT inhibitor. (a) Electrode 1 (E1), (b) Electrode 2 (E2), (c) Electrode 3 (E3). (a-c) reflect three independent cell preparations and five sequential contact experiments per cell. The $(\uparrow)$ arrow indicates the time of contact between the electrode and cell plasma membrane. (d) Cluster plot showing all electrode responses. (e) Bar graph constructed for each electrode, error bars (mean $\pm \mathrm{SD}$ ) reflect five replicated contacts shown in a-c. 

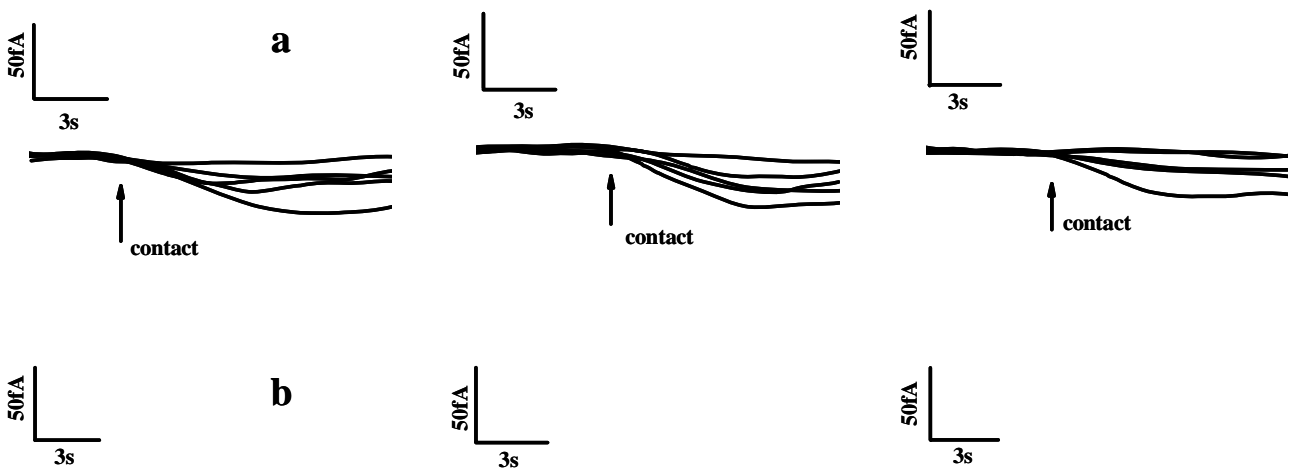

b
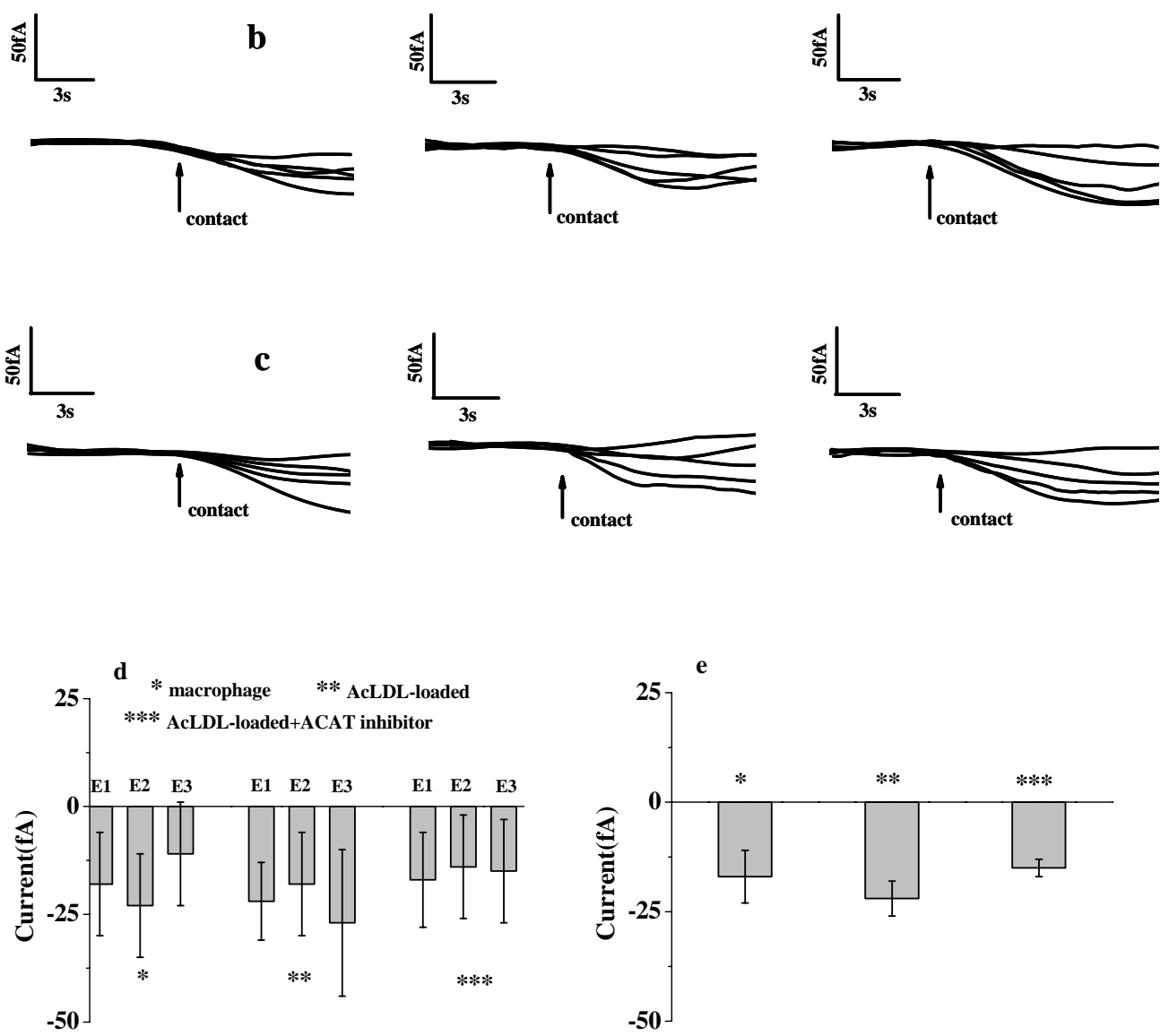

Supporting Figure 4. Control experiments for contacting the three different cell types with three bare Pt electrodes (E1, E2 and E3) containing no immobilized enzyme. a) RAW 264.7 macrophage b) AcLDL-loaded RAW264.7 macrophage and c) AcLDL-loaded RAW264.7 macrophage treated with an ACAT inhibitor. (a-c) reflect three independent cell preparations and five sequential contact experiments per cell. The $(\uparrow)$ arrow indicates the time of contact between the electrode and cell plasma membrane. (d) Bar graph constructed for each electrode, error bars (mean $\pm \mathrm{SD}$ ) reflect five replicated contacts shown in a-c. (e) Averaged data from three electrodes, error bars are mean \pm SD. 


\section{Materials and Methods}

Platinum microelectrode fabrication.

Platinum disk microelectrodes $(4 \mu \mathrm{m}$ diameter) are fabricated in-house. The end of platinum wire $(10 \mu \mathrm{m}$ diameter, Goodfellow Inc.) is electrochemically etched to a tip of less than $1 \mu \mathrm{m}$ in diameter. ${ }^{5}$ The etching procedure involves inserting the platinum wire into a $15 \% \mathrm{CaCl}_{2}$ aqueous solution covered with a layer of acetone. The layer of acetone prevents the generation of foam at the liquid/air interface as gas is produced at the platinum wire during electrochemical etching. The etching voltage perturbation is a sinusoidal waveform $(2.5 \mathrm{~V}$ peak to peak) of $60 \mathrm{~Hz}$ applied between the $\mathrm{Pt}$ wire and a counter electrode (large area Pt electrode). Etching is complete when electrical contact between the etched platinum wire and the solution is broken as indicated by termination of bubble production. The sharpened $\mathrm{Pt}$ wire is washed with $\mathrm{H}_{2} \mathrm{O}$, dried in a steam of nitrogen, and inserted into glass capillaries (Kimax-51, Kimble products). The glass capillary is heated in a platinum coil and pulled (using an attached weight) to form a thin insulating layer of glass on the platinum wire. ${ }^{6}$ The tip of the pulled capillary containing the sharpened platinum wire is polished using a beveling machine (WPI, Inc.) to produce a $4 \mu \mathrm{m}$ diameter Pt disk electrode.

Electrode modification

Immediately after polishing, the platinum disk microelectrodes are immersed in a $5 \mathrm{mM}$ hexane solution of 11-mercaptoundecanoic acid (Aldrich Chem. Co.) for $2 \mathrm{~h}$. These reaction conditions produce a sub-monolayer coverage of 11-mercaptoundecanoic acid on the platinum electrode surface. The sub-monolayer modified platinum surface is exposed to a $100 \mathrm{mM}$ sodium phosphate aqueous solution (PBS, pH 7.4) containing $2 \mathrm{mM} \mathrm{EDC} \mathrm{(Sigma} \mathrm{Chem.} \mathrm{Co.)} \mathrm{and} \mathrm{5mM} \mathrm{NHS}$ (Fisher) for 30 minutes for conversion of the carboxylic acid end groups of the sub-monolayer to an NHS ester8. This activated surface is immersed in $1 \mathrm{mg} / \mathrm{ml}$ solution of recombinant cholesterol oxidase (Oriental Yeast Co. Ltd., 42.0 units $/ \mathrm{mg}$ ) for $2 \mathrm{hrs}$ for covalent attachment of the enzyme to the electrode surface. The noise (pike-to-pike) in the electrochemical experiments is dependent on the electrode used and no speculation is offered for this observation. Approximately half of the prepared electrodes exhibit noise (for as collected data prior to digitally smoothing) of $\leq 50 \mathrm{fA}$ and only such electrodes are used in single cell experiments.

Preparation of cholesterol solution and detection

Cholesterol is dissolved in chloroform and dried under nitrogen before preparing the solutions of required concentration. The dried cholesterol is dissolved in an aliquot of PBS containing $1 \%(\mathrm{v} / \mathrm{v})$ Triton X-100 by sonication. Electrode responses to cholesterol solution at $37^{\circ} \mathrm{C}$ are collected in quiet buffer after spiking with aliquots of cholesterol solution. The spiked aliquots are preheated for the $37^{\circ} \mathrm{C}$ experiments. Injection times are controlled manually.

Data Acquisitions

Amperometric measurements are conducted using a two-electrode cell and a voltammeteramperometer (Chem-Clamp, Dagan corp.). The three-pole Bessel filter of the Chem-Clamp is set to $100 \mathrm{~Hz}$. The signal is further processed using a noise-rejecting voltmeter (model $7310 \mathrm{DSP}$, Signal Recovery Inc.) to digitally filter $60-\mathrm{Hz}$ noise and to provide a DC voltage output with a time constant of $0.1 \mathrm{~s}$. Collected data are smoothed using a moving boxcar average (5-20 data points). $\mathrm{An} \mathrm{Ag} / \mathrm{AgCl}$ $(1 \mathrm{M} \mathrm{KCl})$ reference electrode is used for all experiments, and the applied potential is $820 \mathrm{mV}$ versus NHE for all experiments. For electrochemical evaluation of plasma membrane cholesterol, the cells are rinsed with $37^{\circ} \mathrm{C}$ Dulbecco's Phosphate Buffered Saline (D-PBS) (Invitrogen) and kept in D-PBS at $37^{\circ} \mathrm{C}$ during the experiment.

\section{AcLDL-loaded macrophage cells}

RAW264.7 cells are cultured in Dulbecco's Modified Eagle's Medium (DMEM) supplemented with $10 \%$ fetal bovine serum (FBS). On day 1, the cells are plated in $35 \mathrm{~mm}$ dia. Petri dishes. On day 2, the cells are cholesterol loaded by incubation in serum-free DMEM supplemented with $50 \mathrm{mM}$ glucose, $2 \mathrm{mM}$ glutamine, and $0.2 \%$ BSA (DGGB) containing $0.1 \mathrm{mg} / \mathrm{ml}$ AcLDL overnight.

AcLDL-loaded macrophages with inhibited ACAT

AcLDL-loading RAW264.7 cells are treated overnight in DGGB in the presence or absence of $10 \mu \mathrm{g} / \mathrm{ml}$ acyl-coenzyme A: cholesterol O-acyltransferase (ACAT) inhibitor (58-035). 


\section{References}

1. Fung, Y.S.; Wong, Y.Y. Anal. Chem. 2001, 73, 5302-5309.

2. Bokoch, M.P.; Devadoss, A.; Palencsar, M.S.; Burgess, J.D. Anal. Chim. Acta. 2004, 519, 47-55

3. Haynes, M. P.; Phillips, M. C.; Rothblat, G. H. Biochemistry 2000, 39, 4508-4517.

4. Smaby, J.M.; Momsen, M.; Kulkarni, V.S.; Brown, R.E. Biochemistry. 1996, 35, 5696-5704.

5. Zhang, B.; Zhang, Y.H.; White, H.S. Anal. Chem. 204, 76, 6229-6238.

6. Meulemans, A.; Poulain, B.; Baux, G.; Tauc, L.; Henzel, D. Anal. Chem. 1986, 58, 2088-2091. 